\title{
Clinical outcomes in metaphyseal locking plate fixation of distal femur and proximal tibia fracture
}

\author{
Kale S.Y. ${ }^{1}$, Singh S.D. ${ }^{2}$, Padmawar S. ${ }^{3}$, Patel I. ${ }^{4}$, Ahmed S. ${ }^{5}$, Dhar S.B. ${ }^{6}$ \\ ${ }^{1}$ Dr. Sachin Y Kale, Professor, ${ }^{2}$ Dr. Shikhar D Singh, Assistant Professor, ${ }^{3}$ Dr. Shubham Padmawar, Resident, ${ }^{4}$ Dr. Ibad \\ Patel, Resident, ${ }^{5}$ D. Sohrab Ahmed, Resident, ${ }^{6}$ Dr Sanjay B. Dhar, Professor, All authors are affiliated with Department of \\ Orthopedics, DY Patil Medical College, Navi Mumbai, Maharashtra, India.
}

Address for Correspondence: Dr. Shikhar D Singh, Assistant Professor, Department of Orthopedics, DY Patil Medical College, Navi Mumbai, India, Email: drsinghshikhar@gmail.com

\begin{abstract}
Introduction: Fracture of distal femur and proximal tibial are often difficult to treat and associated with many complications. We aimed to assess the clinical and radiological union time and functional outcome in fractures of distal femur and proximal tibia managed by metaphyseal locking plates and to study the complications associated with it. Methodology: This prospective study was conducted from June 2014 to October 2016 in the Department of Orthopedics, Dr. DY Patil Medical College, Hospital and Research Centre, Nerul, Navi Mumbai, using a consecutive sample of 50 patients fulfilling our study eligibility. Detailed clinical history of the patients and operative details were noted. All patients were followed up at 3 and 6 months, and were assessed for pain, range of movement (ROM), functional outcomes, union times and complications. Results: Distal femur fracture were seen in $42 \%$ cases (most common type A2) while proximal tibia fractures were seen in $58 \%$ cases (most common type II). 28.6\% patients showed fracture union by 5 months, $85.74 \%$ showed union by 6 months. Complaint of pain at 3 months was given by $22 \%$ cases and at 6 months by $6 \%$ cases. Delayed, mal and non-union were seen in $12 \%, 4 \%$ and $2 \%$ of cases respectively. As per the Oxford Knee Score, excellent outcome was seen in $86 \%$ cases while good and fair outcome was seen in $8 \%$ and $6 \%$ cases respectively. Conclusion: Metaphyseal locking plates is a superior implant for the fractures around knee with an excellent functional outcome.
\end{abstract}

Keywords: Fracture, Locked plating, Outcome, Femur, Tibia

\section{Introduction}

Fractures around the knee like distal metaphyseal fractures of femur and of proximal tibia have become a common occurrence in present mechanized world. Distal femur fractures have been reported to account for $7 \%$ of all femoral fractures and tibial plateau fractures constitute $1 \%$ of all fractures and $8 \%$ of fractures in the elderly[1].

These fractures are often difficult to treat, and they are associated with many complications [2]. During the past 40 years operative techniques and implants have dramatically improved, and internal fixation is recommended for most distal femoral fractures in adults. Locked plates are a hybrid of plate technology and percutaneous bridge plating using locked screws as a fixed-angle device and have been shown to allow much greater load bearing than regular plates. It has been studied that locking compression plate has the advantage

Manuscript Received: $6^{\text {th }}$ December 2017

Reviewed: $16^{\text {th }}$ December 2017

Author Corrected: $24^{\text {th }}$ December 2017

Accepted for Publication: $31^{\text {st }}$ December 2017 of combination of conventional compression plating and locked plating techniques which enhances the plate osteosynthesis. They also provide adequate load-bearing strength to avoid medial and lateral plating in the distal femur, proximal tibia, and tibial plateau. Additionally, a locking compression plate has got distinct advantages of unicortical fixation and least chance of plate back out as the screw gets locked to the plate. The present study aimed to assess the average clinical and radiological union time and functional outcome in patients with fractures of distal femur and proximal tibia managed by metaphyseal locking plates and also to study the complications associated with it.

\section{Methodology}

Type and place of study- This prospective study was conducted from June 2014 to October 2016 in the Department of Orthopedics, Dr. DY Patil Medical College, Hospital and Research Centre, Nerul, Navi 


\section{Original Research Article}

Mumbai, after obtaining adequate permission from the Institutional Ethics Committee. All patients were explained the purpose and rationale of the study and written informed consent was obtained from all the patients prior to their enrolment in the study.

Sampling technique and inclusion/exclusion criteriaUsing consecutive type of non-probability sampling a total of 50 patients who were scheduled to undergo surgery for fractures around knee i.e. distal femur and proximal tibia fractures were taken in the study after informed consent. We included patients aged 18 years and above of either sex, with closed and open fractures of distal femur or proximal tibia. We excluded patientswithpathological fracture, middle shaft or segmental fractures, with severe systemic illness (active cancer, chemotherapy, haemophilia, or medical contraindication for surgery) or non-consenting patients.

Study definitions- Patients were evaluated for subjective outcomes like pain, functional limitations and occupational considerations, objective outcomes like inspection of the knee joint for deformity, tenderness, abnormal mobility, any previous scare marks around knee, discharging sinus or any other signs of infection. Functional evaluation was performed for range of movements, stiffness of knee, pain while doing routine activities and ability to do day to day activities.

The fractures were classified according to the AO/OTA classification for distal femur and Schatzker classification for proximal tibia. For evaluating range of motion (ROM) stiffness or significant loss of knee range of motion was defined as flexion less than 90 degree [3].

The tibial plateau angle on anteroposterior radiographs and the posterior slope angle on lateral radiographs were measured; tibial plateau angle $>90$ or $<80$ degrees or posterior slope angle $>15$ or $<-5$ degrees was defined as malalignment. An increase of $2 \mathrm{~mm}$ of intra-articular stepoff was defined as secondary loss of reduction.

An intra-articular step-off of at least $5 \mathrm{~mm}$ measured on radiographs was defined as malreduction. Cortical union of at least 3 cortices was defined as bony union and no clinical or radiological evidence of bone healing after 9 months was defined as non-union. Patient assessed for fracture union and knee joint range of motion. The earliest radiographic sign of union on plain radiographs was taken as bridging of the fracture site by callus, crossing/bridging of the fracture site by trabeculae, cortical union of at least 3 cortices or disappearance of fracture line on plain radiograph. Functional outcome was evaluated according to the Oxford Knee Score (OKS) criteria and was graded as poor (0-19), fair (20-29), good (30-39) and excellent (40-48) [4].

Data Collection- A detailed clinical history of the patients was taken. Results of various imaging techniques were noted. Computed Tomography scan of distal femur or proximal tibia was done to see the exact size and number of fragments and to plan the operative fixation. All other patients were operated for surgery as soon as possible depending on their medical condition, skin condition around fracture, and the amount of swelling.

All surgeries were done under $\mathrm{C}$-arm image intensifier control. For tibial fractures antero-lateral approach for the lateral plate and medial or postero-medial approach for the second plate was done. And for femur lateral approach was used. All the patients were managed by locking plates. Length of plate was determined intraoperatively after fracture reduction. Bone grafting was done if the need to fill the voids after reduction was felt.

Post-operative radiographs were taken to assess adequacy of articular reduction, plate position, metaphyseodiaphyseal reduction, and alignment. Active knee motion was encouraged on the 3rd day after surgery. The patients were discharged after an average 90 degrees of range of motion was obtained.

ROM was assessed at 3 and 6 months. All patients were followed up in the outpatient clinic, and were assessed for radiological and clinical union and any post-operative complications.

Statistical analysis- We described the baseline characteristics of the study participants using descriptive statistics. Tests of significance were decided after applying tests of normality. Categorical data were analysed using chi-square. Parametric correlation analysis was done using Pearson correlation test while non parametric correlation analysis were done using Spearman correlation test.

\section{Results}

During the study period we included 50 patients. Mean age of the study patients was 43.4 years with almost half of the subjects were between 41-60 years of age (Table 1). 
Table-1: Baseline characteristics of the patients included in the study.

\begin{tabular}{|c|c|}
\hline Variables & Numbers \\
\hline \multicolumn{2}{|l|}{ Age distribution } \\
\hline $21-30$ years & 13 \\
\hline $31-40$ years & 6 \\
\hline $41-50$ years & 17 \\
\hline $51-60$ years & 7 \\
\hline $61-70$ years & 7 \\
\hline \multicolumn{2}{|l|}{ Gender distribution } \\
\hline Females & 14 \\
\hline Males & 36 \\
\hline \multicolumn{2}{|l|}{ Site of fracture } \\
\hline Distal femur & 21 \\
\hline Proximal tibia & 29 \\
\hline \multicolumn{2}{|l|}{ Mode of injury } \\
\hline Fall & 24 \\
\hline Road traffic accident & 26 \\
\hline \multicolumn{2}{|l|}{ Compound fracture } \\
\hline Closed & 41 \\
\hline Compound & 9 \\
\hline \multicolumn{2}{|l|}{ Plating technique used } \\
\hline Minimally invasive plate osteosynthesis & 4 \\
\hline Open & 46 \\
\hline Use of bone graft & 12 \\
\hline \multicolumn{2}{|l|}{ Bridging of fracture on X-ray } \\
\hline 2 months & 23 \\
\hline 3 months & 26 \\
\hline
\end{tabular}

Male predominance was observed in the study with $72 \%$ males. Distal femur fracture were seen in $42 \%$ cases while proximal tibia fractures were seen in $58 \%$ cases and most common mode of injury was road traffic accident (52\%) followed by fall $(48 \%)$.

In $46 \%$ cases, bridging of fracture site on x-ray was seen at 2 months ( 8 weeks) while in $52 \%$ cases it is visible at 3 months (12 weeks).

Most common type of femur fracture according to AO classification was type A2 (Table 2). The overall prevalence of type A, $\mathrm{B}$ and $\mathrm{C}$ fractures were $57.1 \%, 9.6 \%$ and $33.4 \%$ respectively.

Most common type of tibia fracture according to Schatzker classification was type II (34.5\%) followed by type 5 and 6 (20.7\% each). Distal femur fractures were associated with proximal tibia fractures in 3 cases while tibia shaft fracture was seen in 2 cases (Table 2). 
Table-2: Operative details of the patients included in the study.

\begin{tabular}{|c|c|c|c|}
\hline Femur fracture & & Tibia fracture & \\
\hline Variables & Number & Variables & Number \\
\hline \multicolumn{2}{|l|}{ Classification of fracture (AO type) } & \multicolumn{2}{|c|}{ Classification of fracture (Schatzkar type) } \\
\hline $\mathrm{A} 1$ & 2 & Type 1 & 3 \\
\hline A2 & 7 & Type 2 & 10 \\
\hline A3 & 3 & Type 3 & 4 \\
\hline $\mathrm{B} 1$ & 1 & Type 5 & 6 \\
\hline B2 & 1 & Type 6 & 6 \\
\hline $\mathrm{C} 1$ & 3 & & \\
\hline $\mathrm{C} 2$ & 3 & & \\
\hline C3 & 1 & & \\
\hline Type of plate used & & Type of plate used & \\
\hline Distal femur anatomical locking plate & 7 & Anatomical locking bicondylar plate & 5 \\
\hline \multirow[t]{4}{*}{ Distal femur locking plate } & 14 & Bicondylar prox tibia locking plate & 1 \\
\hline & & $\begin{array}{l}\text { Proximal tibia anatomic locking } \\
\text { plate }\end{array}$ & 6 \\
\hline & & Proximal tibia locking plate & 16 \\
\hline & & Raft locking plate & 1 \\
\hline Associated injuries & & Associated injuries & \\
\hline Fibullar fracture & 1 & Distal femur + fibular fracture & 1 \\
\hline Lateral malleolar fracture & 1 & Distal femur fracture & 2 \\
\hline Proximal tibial fracture & 3 & Fibular fracture & 7 \\
\hline Tibial shaft fracture & 2 & Lateral collateral ligament sprain & 1 \\
\hline None & 14 & None & 18 \\
\hline Time to surgery & & Time to surgery & \\
\hline $0-5$ days & 11 & & 7 \\
\hline 6-10 days & 7 & & 16 \\
\hline 11-15 days & 2 & & 5 \\
\hline 16-20 days & 1 & & 1 \\
\hline
\end{tabular}

After the fracture, days elapsed before surgery in distal femur was 0 to 5 days in most cases and in proximal tibia it was 6 to10 days in most cases. The mean time elapsed before surgery was 5.38 days with minimum of 1 day to maximum of 20 days.

A total of $28.6 \%$ patients showed fracture union by 5 months (20 weeks), $85.74 \%$ showed union by 6 months (24 weeks). Complaint of pain at 3 months was given by $11(22 \%)$ cases while at the end of 6 months pain was experienced in only 3 $(6 \%)$ cases (Table 3$)$.

Range of motion (ROM) less than 90 degrees at 3rd month was seen in 14\% cases while 42.5\% had ROM over 110 degrees. The range of motion is increased by 6 months. Delayed, mal and non-union was seen in $12 \%, 4 \%$ and $2 \%$ of cases respectively. As per the Oxford Knee Score, excellent outcome was seen in $86 \%$ cases while good and fair outcome was seen in $8 \%$ and $6 \%$ cases respectively. 
Table-3: Post-operative details of the patients included in the study.

\begin{tabular}{|c|c|}
\hline Variables & Number \\
\hline \multicolumn{2}{|l|}{ Fracture union time } \\
\hline Less than 5 months & 14 \\
\hline More than 5 months & 35 \\
\hline \multicolumn{2}{|c|}{ Complaints of postoperative pain } \\
\hline At 3 months & 11 \\
\hline At 6 months & 3 \\
\hline \multicolumn{2}{|c|}{ Range of motion at 3 months } \\
\hline Less than 90 degrees & 7 \\
\hline 90 to 110 degrees & 22 \\
\hline More than 110 degrees & 21 \\
\hline \multicolumn{2}{|c|}{ Range of motion at 6 months } \\
\hline Less than 90 degrees & 3 \\
\hline 90 to 110 degrees & 6 \\
\hline More than 110 degrees & 41 \\
\hline \multicolumn{2}{|c|}{ Postoperative complications } \\
\hline Delayed union & 6 \\
\hline Malunion & 2 \\
\hline Nonunion & 1 \\
\hline Knee stiffness & 3 \\
\hline Surgical wound infection & 3 \\
\hline Osteomyelitis & 1 \\
\hline None & 42 \\
\hline \multicolumn{2}{|c|}{ Functional outcome (Oxford Knee Score) } \\
\hline Excellent & 43 \\
\hline Good & 4 \\
\hline Fair & 3 \\
\hline Poor & 0 \\
\hline
\end{tabular}

\section{Discussion}

Present hospital based prospective observational study was conducted with the objective of studying the average clinical and radiological union time in fractures of distal femur and proximal tibia managed by metaphyseal locking plates and also to observe associated complications. Male predominance of patients included in our study, which can be attributed to more outdoor activities by males and thus being more prone to vehicular accident and falls.

Looking at the classification of fractures in our study, we can comment that most of the fractures around the knee are due to high energy trauma and sometimes are

Surgical Update: International Journal of Surgery \& Orthopedics associated with severe comminution. The relatively lower incidence of AO type-3 fractures of distal femur and Schatzker type- 6 fractures of proximal tibia can be attributed to relatively higher percentage of fall injuries encountered in our study as compared to other studies where road traffic accident (RTA) were the major cause of injury.

In our study population, the mean time to surgery was 5.38 days ranging from 1 day to 20 days. In a study by Singh et al, majority of the surgeries (54\%) were performed within the first 48 hours of injury, with a mean time to surgery of 4.12 days [5]. In a study by Gupta et al

Available online at: www.surgicalreview.in 154 | P a g e 


\section{Original Research Article}

most of the patients were operated for distal femur within 7 days after injury [6].In a similar study on 100 distal femoral fractures, Gupta VK et alError! Bookmark not defined. concluded that early surgery, at least two screws in each fragment and early post-operative knee mobilization are essential for good union and good knee range of motion. Furthermore, Kyle $\mathrm{F}$ et al recommended locking plates in large aging population for continuing active lifestyles [7]. If used appropriately, locked plating can result in good patient outcomes with recreation of bony architecture and restoration of function.Tan et alproposed that locked plating has evolved simultaneously with the minimally invasive techniques, which together provide an elegant safe option for restoring function in well trained, experienced hands [8].

In present study, bone graft, which was used in $24 \%$ of the cases, was used to fill the void after reduction mostly in fracture in osteoporotic bone or comminuted fractures like Schatzker type 2,3,5,6 and distal femur AO type A2,A3,C2,C3. ROM less than 90 degrees at 3rd month was seen in $14 \%$ cases while $42.5 \%$ had ROM over 110 degrees. Range of motion (ROM) less than 90 degrees at 6 th month was seen in $6 \%$ cases while $82 \%$ had ROM over 110 degrees.Kanabar et al in their series of 17 patients operated with minimally invasive plate osteosynthesis (MIPO) or Less Invasive Stabilization System (LISS) of distal femur reported average range of motion of 0 to 100 degrees [9].Lee et al in their series of 35 patients of proximal tibial fractures operated with MIPO reported an average range of motion of 105 degrees [10].In addition, Collinge et al had evaluated the results of treatment of complex tibial periarticular fractures using percutaneous technique in seventeen patients and found that the range of motion in patient with open fractures averaged 5-degree full extension to 130-degree flexion [11]. Obtaining full range of movements depends mainly on starting early ROM exercises in stable fixation of fracture. While previous authors have reported functional outcomes using various scales like Rasmussen clinical score and Neer criteria, we used the Oxford Knee Score (OKS) and found it to be excellent in $86 \%$ cases while good and fair outcome was seen in $8 \%$ and $6 \%$ cases respectively.

What this study adds to existing knowledge- Very few studies have been done previously of locking compression plates that include both distal femoral and proximal tibial fractures. Our study shows that minimum periosteal stripping preserves blood supply and fracture haematoma is not disturbed which promotes faster healing. Locking compression plates have excellent outcome for fractures around the knee and knee range of motion is excellent with the use of this implant.

\section{Conclusion}

On the basis of these observations, we conclude that metaphyseal locking plates is a superior implant for the fractures around knee i.e. distal femur and proximal tibia with an excellent functional outcome. The locking plates preserve the periosteal blood supply and hence the union is early as compared to other methods. With MIPO technique less periosteal stripping, preservation of fracture haematoma, and less wound related complications were observed.

All these factors when combined with a stable fixation give the bone a near anatomical environment to heal and this results in lesser complications, early union and better functional outcome.

Author contributions: SYK conceptualized the study, helped with manuscript writing and checked the manuscript for scientific content. SDS helped with manuscript writing, data collection and analysis and takes the overall responsibility of the manuscript. SP, IP and SA helped with data collection. SBD helped with study design and manuscript writing.

Conflict of interest: None declared.

Funding: Nil, Permission from IRB: Yes

\section{References}

1. Phisitkul P, McKinley TO, Nepola JV, Marsh JL. Complications of locking plate fixation in complex proximal tibia injuries.J Orthop Trauma. 2007 Feb;21 (2): 83-91.

2. Kiran KG, Sharma G, Farooque K, Sharma V, Ratan R, Yadav S, Lakhotia D. Locking Compression Plate in Distal Femoral Intra-Articular Fractures: Our Experience. International scholarly research notices. 2014:372916.

3. Jain R. Prospective Case Study of Outcome of Tibial Plateau Fractures Treated with Locking Condylar Plate. Malaysian orthopaedic journal. 2016;10(3):12.

4. Khatri K, Lakhotia D, Sharma V, GN KK, Sharma G, Farooque K. Functional Evaluation in High Energy (Schatzker Type V and Type VI) Tibial Plateau Fractures Treated by Open Reduction and Internal Fixation. International scholarly research notices. 2014:589538-.

5. Singh SK, Kishore N, Singh A, Nag S, Hembram S. A Comparative Study-Plating Of Fracture Around Knee Joint By Mipo V/S Conventional Technique. IOSR Journal of Dental and Medical Sciences. 2015;14(1): $37-47$. 


\section{Original Research Article}

6. Gupta SV, Dande R. Surgical management of fracture of distal end of femur in adults by minimal invasive percutaneous plate osteosynthesis (MIPPO) with locking condylar plate. International Journal of Orthopaedics. 2015;1(2):07-11.

7. Dickson KF, Munz J. Locked plating: clinical indications. Techniques in Orthopaedics. 2007;22(3): $181-5$.

8. Tan SL, Balogh ZJ. Indications and limitations of locked plating.Injury. 2009 Jul;40(7):683-91. doi: 10.1016/j.injury.2009.01.003. Epub 2009 May 22.
9. Kanabar P, Kumar V, Owen PJ, Rushton N.Less invasive stabilisation system plating for distal femoral fractures.J Orthop Surg (Hong Kong). 2007 Dec;15(3): 299-302.

10. Lee JA, Papadakis SA, Moon C, Zalavras CG. Tibial plateau fractures treated with the less invasive stabilisation system. International orthopaedics. 2007;31 (3):415-8.

11. Collinge C, Sanders R, DiPasquale T. Treatment of complex tibial periarticular fractures using percutaneous techniques. Clinical orthopaedics and related research. 2000;375:69-77.

\section{How to cite this article?}

Kale S.Y, Singh S.D, Padmawar S, Patel I, Ahmed S, Dhar S.B. Clinical outcomes in metaphyseal locking plate fixation of distal femur and proximal tibia fracture. Surgical Update: Int J surg Orthopedics.2017;3(4):150-156.doi:10. 17511/ijoso.2017.i04.10. 